\title{
Entre bebês, abismos e fantasmas: narrativas para pensar a relação entre clínica e saúde coletiva*1
}

\author{
Cláudia Odiléia Müller*2 \\ Analice de Lima Palombini*3
}

$O$ artigo propõe uma reflexão sobre a relação entre Clínica e Saúde Coletiva a partir de narrativas de casos clínicos de bebês atendidos com aporte teórico da psicanálise no âmbito da Atenção Básica do Sistema Único de Saúde. Observou-se que, no encontro entre os saberes dos usuários com os saberes técnicos das disciplinas envolvidas e com os saberes da Saúde Coletiva, acontece um movimento que circula em uma superfície paradoxal, produzindo uma relação de "suplementaridade moebiana” entre clínica e Saúde Coletiva.

Palavras-chave: Clínica, saúde coletiva, primeira infância, psicanálise

*1 Este artigo é referente a uma pesquisa de mestrado realizada no Programa de Pós-Graduação de Psicologia Social e Institucional da Universidade Federal do Rio Grande do Sul (PPGPSI/UFRGS). A banca de defesa da dissertação ocorreu em 27/6/2014 e este artigo faz parte da exigência acadêmica para a homologação do título de mestre. O título da dissertação é "Entre bebês, abismos e fantasmas: narrativas sobre uma clínica em Saúde Coletiva".

*2, ${ }^{3}$ Universidade Federal do Rio Grande do Sul - UFRGS (Porto Alegre, RS, Br). 


\section{Introdução}

A Saúde Coletiva caracteriza-se pela multicausalidade do processo de saúde e de doença, em contraposição ao saber hegemônico do paradigma biomédico. A discussão sobre qual clínica se exerce desde este paradigma marca, desde o início, a pluralidade de arranjos entre os diferentes saberes técnicos, os poderes por eles exercidos e os saberes e poderes políticos e administrativos. Em vez de uma pragmática única, tem-se a multiplicidade de fazeres e de saberes sobre saúde habitando o mesmo campo, no qual os limites são imprecisos, em constante processo de elaboração (Birman, 2005; Luz, 2009; Nunes et al., 2010; Paim \& Almeida, 1998).

A multiplicidade, característica própria do humano, afeta, de igual modo, o que se entende como saúde e como adoecer, marcado pelos processos de determinação social - processos socioeconômicos, ecológicos, biológicos, psicológicos e culturais, articulados às dimensões políticas, históricas e espaço-territoriais que produzem saúde e doenças (Tambellini \& Schütz, 2009).

Assumindo como premissa a determinação social do processo saúde e doença (Campos, 2005), a Saúde Coletiva voltou seus esforços para a inclusão da instância do coletivo, em contraposição à do risco individual da doença calculado pela epidemiologia clássica, característica da clínica tradicional instituída na Saúde Pública. Contemplou-se, desde então, o desenvolvimento de atividades de investigação sobre o estado sanitário da população, a natureza das políticas de saúde, a relação entre os processos de trabalho e doenças e agravos, bem como as intervenções de grupos e classes sociais sobre a questão sanitária. A epidemiologia, o planejamento/administração de saúde e as ciências sociais em saúde, de acordo com Paim \& Almeida (1998), foram consideradas as disciplinas básicas da Saúde Coletiva, configurando um campo de conhecimento interdisciplinar. A clínica, juntamente com a genética, as ciências biomédicas, entre outras, seriam disciplinas complementares desse campo. 
A Saúde Coletiva, como campo de conhecimentos interdisciplinares, fundamenta um âmbito de práticas transdisciplinar, multiprofissional, interinstitucional e transetorial (Paim \& Almeida, 1998), cujas práticas tomam como objeto as necessidades sociais de saúde, centrando suas intervenções nos grupos sociais e no ambiente. A Vigilância em Saúde é uma de suas principais formas de intervenção na realidade sanitária, utilizando métodos de promoção e prevenção para agir no território, nas instituições e nas coletividades (Campos, 2005).

Tamanha ênfase no coletivo provocou um efeito colateral: o recalque da categoria clínica no campo da Saúde Coletiva (Onocko-Campos, 2012). A clínica carregaria a marca das práticas individualizantes, herdeiras da medicina tradicional cujo foco estaria na doença, em uma abordagem exclusivamente biológica do adoecer, sem considerar seu caráter histórico e social. A crítica aos especialismos, responsáveis pela fragmentação das ações de saúde, também contribuiu neste processo, levando à banalização da técnica. Tal banalização pode dificultar a percepção das diferenças com as quais os trabalhadores em saúde comparecem ao encontro com o usuário. Afinal, é sempre de um encontro que se trata, no qual se produz saúde com e para pessoas que singularizam modos de subjetivação próprios de seu tempo.

É sobre este encontro que versa este trabalho. Um encontro no âmbito da 294 Atenção Básica do Sistema Único de Saúde (SUS), entre saberes disciplinares advindos dos profissionais da nutrição e da psicologia — tendo a psicanálise como aporte teórico das práticas narradas - e os saberes dos usuários. São saberes que transitam entre as dimensões conscientes e inconscientes, próprias do humano, em um encontro no qual afetos estão em contato, configurando um jogo de resultado sempre incerto (Onocko-Campos, 2012).

À clínica sob recalque na Saúde Coletiva, aproxima-se esse outro saber que também enfrenta resistências neste campo: a psicanálise. Identificada ao setting clínico convencional, a psicanálise tradicionalmente é tida como prática elitista, voltada para o individual, para sua interioridade. "A" psicanálise é criticada pelos ditos pós-estruturalistas em relação ao pressuposto de universalidade, presente em muitas das suas conceituações; em relação à afirmação de uma essência subjetiva, na qual o inconsciente toma um lugar central; e em relação à noção de permanência estrutural do sujeito e da psicopatologia (Torossian, 2007). Tais críticas e resistências contribuíram para que, na Saúde Coletiva, apesar do resgate do sujeito em suas dimensões histórica, social e coletiva, o sujeito do inconsciente tenha sido "varrido em uma maré de boas intenções", junto com repertórios teóricos e habilidades técnicas, no furor da crítica aos especialismos (Onocko-Campos, 2012, p. 13).

Mas existem muitas psicanálises, muitos autores e vertentes teóricas referenciados à instância do inconsciente. Ricardo Rodulfo (2008) é um dos que tomou as críticas à psicanálise como objeto de análise e, a partir da própria clínica, 


\section{SAÚDE MENTAL}

empreendeu a desconstrução do texto psicanalítico conforme a proposta de Jacques Derrida (1997) — não para desprezar a psicanálise, mas, ao contrário, para permitir-lhe ir além das oposições binárias que conformam e armazenam as teorias dentro de seus campos.

A psicanálise aqui comparece para oferecer à Saúde Coletiva uma noção de sujeito que transcende a ideia de indivíduo, apresentando-se não como lugar da verdade, mas como lugar de seu desconhecimento (Broide, 2009). A partir desta constatação, ou seja, de que ao sujeito cabe algo sobre si mesmo que ele não sabe, a Saúde Coletiva pode potencializar dispositivos institucionais capazes de viabilizar o que só se consegue expressar pela via do sintoma. Além disso, reconhecer a instância do inconsciente muda a forma como se planeja e executa o trabalho em equipes, o trabalho da gestão e com coletivos (Figueiredo, 2005; Onocko-Campos, 2012; Palombini et al., 2011).

Isso posto, cabe, então, a pergunta: Como vem se constituindo a prática clínica na Saúde Coletiva?

Acreditamos que lançar o olhar sobre a clínica que se produz no cotidiano do trabalho do SUS pode ajudar a pensar uma clínica na Saúde Coletiva. Assim, investiu-se na narrativa da experiência clínica como forma de constituir um saber advindo da prática para contribuir na construção do território do SUS pelos seus trabalhadores. Foram elaboradas duas narrativas de atendimentos clínicos de bebês conduzidos por uma equipe de nutrição e de psicologia - a qual tomou a psicanálise como referência teórica e ética do trabalho. A partir da experiência da clínica entre saberes diversos no atendimento com bebês no âmbito do SUS, empreendeu-se o trabalho de investigar o que a clínica com bebês poderia fazer operar na construção de conhecimentos sobre a clínica na Saúde Coletiva. ${ }^{1}$

As narrativas foram elaboradas por um coletivo do qual participaram como pesquisadoras-colaboradoras duas nutricionistas e uma ex-estagiária de psicologia, as quais, juntamente com a mestranda, atenderam aos casos clínicos narrados acolhidos pelo Nutrir. ${ }^{2}$ Walter Benjamin (2012) e Ricardo Rodulfo (2008) são inspiradores das narrativas. Benjamin, por conceber, com o seu narrador sucateiro, uma narrativa articulada de forma inextrincável à transmissão de uma experiência. E Rodulfo, por elaborar uma forma de transmitir a experiência psicanalítica por

${ }^{1}$ A pesquisa de mestrado da qual se origina este artigo respeitou os aspectos éticos e legais implicados no trabalho com pessoas, com aprovação pelo Comitê de Ética em Pesquisa sob parecer de n. $480.373 / 2014$.

${ }^{2}$ O Nutrir é um Centro de Educação Nutricional, que realiza a coordenação técnica do serviço de nutrição da Secretaria Municipal de Saúde da Prefeitura Municipal de Novo Hamburgo/RS e atua nos eixos da atenção, gestão e educação em nutrição. 
meio de um modelo clínico e narrativo extraído diretamente da prática, nominado por ele de Estudo Clínico. Segundo o autor, como um gênero de pesquisa, o Estudo Clínico focaliza um processamento de materiais não assimilável à aplicação de um molde sobre uma massa, mas ao processo do amassar, do qual irá surgindo a conceitualização.

Apresentaremos a seguir duas categorias de análise colhidas do material das narrativas, a partir das quais se questiona a relação entre clínica e Saúde Coletiva.

\section{Sucatas e extratos}

O narrador benjaminiano coletou sucatas, restos, os detalhes rememorados das vivências, para elaborar as narrativas como atitude ético-política de não deixar cair em esquecimento o que se viveu no Nutrir. Mas este narrador, acompanhado de Rodulfo, queria brincar. Ele também estava interessado em poder dar lugar a alguns questionamentos que a prática fez emergir, questionamentos que nem sequer estavam elaborados na forma de perguntas; era uma suspeita de que algo ali precisava ser mais remexido, para extrair os restos, para elencar significantes com os quais se pudesse constituir uma experiência.

Um olhar sobre as duas narrativas produzidas, uma sobre Luiza ${ }^{3}$ e outra sobre Luís André, ambos os bebês atendidos pela equipe multiprofissional de nutrição e psicologia, pode destacar alguns recortes que, de algum modo, em ambas as narrativas, marcam uma presença que se repete. Diferentes sucatas, diferentes lembranças, foram nomeadas pelos mesmos significantes, que dizem respeito ao modo como se constituiu a clínica entre saberes nas experiências narradas. "Encruzilhada clínica" e "Encantamentos" são significantes que se referem a categorias de análises possíveis ao material contido nas narrativas, sobre as quais se faz necessário refletir.

\section{Encruzilhada clínica}

Encruzilhada é um ponto onde se cruzam vários caminhos. Há sempre uma tensão quando se chega a ela. Para onde seguir? Qual escolha fazer? Quais critérios usar para fazer escolhas?

${ }^{3}$ As narrativas completas encontram-se na dissertação que deu origem a este artigo, no link: $<$ http://www.lume.ufrgs.br/handle/10183/115073>; 


\section{SAÚDE MENTAL}

No caso de Luiza, a encruzilhada se evidencia quando esta não consegue nem recuperar o peso que tinha ao nascer, nem continuar crescendo. Mas a encruzilhada é realmente tensionada no momento em que Luiza - sem a presença de qualquer complicação orgânica em seu desenvolvimento - achava-se no limiar de peso para uma internação hospitalar. No caso de Luís André, ela se anuncia no tempo em que nutricionista e psicóloga que atendem o caso, percebem o atraso do desenvolvimento enquanto são surpreendidas pelo relato de violência e de loucura da mãe em meio a um quadro de vulnerabilidade social. Mas a encruzilhada de fato se tensiona no momento em que várias vozes clamam pelo abrigamento do bebê. Ao longo dos dois casos, há vários momentos nos quais este tensionamento retorna $\mathrm{e}$ precisa ser encarado pela equipe e pela rede intersetorial de atendimento. Os tensionamentos indicam necessidade de tomar posição, de fazer escolhas e, muitas vezes, de deflagrar lutas para sustentá-las.

Especialmente em casos que envolvem bebês, a encruzilhada clínica se intensifica com a dimensão da temporalidade que, por vezes, se torna urgência. A fragilidade e a dependência do bebê humano, assim como as características próprias do desenvolvimento neuropsicomotor, impõem uma implicação temporal às intervenções clínicas. Temporalidade que Julieta Jerusalinsky (2002) tomou em análise, nominando três aspectos de sua constituição: 1) O "tempo real" - aquele que determina certa janela temporal para uma aquisição no desenvolvimento do bebê; 2) A "temporalidade imaginária" — referida a uma normatização cronológica do desenvolvimento a partir da existência de um tempo real que marca uma correlação entre a idade e as aquisições esperadas naquele período, antecipando imaginariamente o que se espera e o que se demanda para a criança em determinado espaço-tempo do desenvolvimento; 3) A "temporalidade simbólica" — referida ao circuito de desejo e demanda no qual o bebê é tomado desde o campo do Outro e que instaura "tempos lógicos" nos quais os eventos psíquicos são inscritos e reinscritos. A articulação destes três aspectos da temporalidade engendra o bebê como sujeito do desejo e como autor das aquisições instrumentais de seu desenvolvimento.

Em ambos os casos narrados, uma delicadeza colocada em pauta consistia em inventar formas de intervir que não desarticulassem os três registros temporais. No caso de Luiza, vemos como a temporalidade real implicava uma urgência, na medida em que a janela temporal se fechava e ela não adquiria peso que pudesse sustentar seu crescimento. É quando se torna imperativo avaliar a necessidade de uma prescrição, como a internação hospitalar, para instalar uma descontinuidade no processo e provocar uma nova bifurcação no caminho.

Passada a etapa emergencial, quando Luiza finalmente começou a ganhar peso, os atendimentos puderam seguir um rumo menos prescritivo. Sim, uma dose de prescrição é necessária quando uma vida está em risco, mas ela não se sustenta sem uma dose muito delicada de escuta clínica. Se isso não funcionar, então é 
necessário usar de recursos invasivos — como uma internação hospitalar. Estamos falando de intervenções em casos graves, nos quais o risco de vida é eminente.

No caso de Luís André, o registro do tempo real poderia estar comprometido devido às suas limitações orgânicas que, apesar de não completamente esclarecidas pela inconclusão do diagnóstico clínico, estavam presentes. Mas o que se fazia necessário era muito mais do que observar como se articulavam o tempo imaginário e o simbólico, apesar da defasagem do tempo real. Era necessário que as equipes e a rede de atendimento construída para atendê-lo se comprometessem a promover a articulação destes registros, criando um projeto terapêutico singular que levasse em consideração a situação de vida da família na avaliação da "encruzilhada clínica". Felizmente foi possível contar com um trabalho interdisciplinar de estimulação precoce, a partir do corte epistemológico da psicanálise, implicado na clínica no sentido de produzir um enlaçamento entre um real orgânico e uma tela simbólica (Jerusalinsky, 2002). A delicadeza estava em avaliar, no andar do processo, o quanto os registros simbólicos e imaginários estavam ativos e provocativos da emergência de um sujeito, o qual tinha limites ao mostrar sua atividade nas relações.

Se não fosse possível sustentar a proposta de articulação entre os registros temporais juntamente com os pais e/ou familiares do bebê, então seria necessário uma intervenção do Estado, de que este não poderia se eximir. Sim, em situações extremas o Estado deve abrigar bebês, mas somente para oferecer um espaço onde seja possível que o desejo pelo bebê se constitua, e não unicamente para promover uma separação entre o bebê e seus pais "inadequados". Este é o dilema do abrigamento de um bebê: o fato da constituição subjetiva ser ainda tão precária, dependente, e o quão singular é o laço de desejo que inscreve o bebê na linguagem. Por isso deve-se apostar em ações intersetoriais, que oportunizem para os pais uma nova experiência em relação ao seu bebê, sempre que isto for possível. Infelizmente, nem sempre é. A cada caso, deve ser detalhadamente avaliado o risco e o benefício de fazer um abrigamento. Quando abrigamos bebês que têm dificuldade de serem ativos e provocativos na relação com o outro, como é o caso de bebês com atraso no desenvolvimento, é grande o risco de se produzir uma sobrevida biológica, que reduz o homem à sua condição residual, não humana.

Quando se trata de bebês, percebe-se de forma muito clara o quanto é importante os técnicos compreenderem as questões envolvidas na temporalidade em relação ao desenvolvimento psíquico e orgânico, que estão interligadas de forma inextrincável. $\mathrm{O}$ atendimento de bebês dá visibilidade à “encruzilhada clínica". Mas há também outra dimensão na encruzilhada, tão fundamental quanto os saberes técnicos: a dimensão da vida cotidiana das famílias envolvidas, considerando a vulnerabilidade social, dimensão na qual os saberes da Saúde Coletiva são fundamentais para a elaboração de um projeto terapêutico singular, como no caso de Luís André. Compreender os fatores históricos e sociais do território em que vivem, 


\section{SAÚDE MENTAL}

entender como se articulam os dispositivos públicos e os das comunidades, quais espaços podem ser potencializados para o cuidado da família, como se enlaçam as ações intersetoriais, são saberes oferecidos pela Saúde Coletiva, que acompanham a caminhada e se encontram com os saberes técnicos na encruzilhada.

Assim, há que se fazer um "trabalho forte" quando se chega a uma encruzilhada. A tradição cultural afro-brasileira já nos ensinou isso há muito tempo. É necessário reconhecer e analisar cada caminho que leva à encruzilhada para fazer escolhas por onde seguir. Tal trabalho exige "encantamentos" e muitos encontros para discutir casos, ou construí-los, como propõe Figueiredo (2004).

Note-se que a encruzilhada clínica só é percebida por quem já está disposto a bifurcar caminhos, por quem fez uma escolha ético-política pela multiplicidade de caminhos possíveis para se produzir saúde, por quem se recusa a tomar sujeitos como objetos das ações dos especialistas. Esta escolha aponta para o fato de que não são os saberes a referência primeira para uma intervenção clínica, social e política. É a ética que referencia as escolhas - ética entendida como a problematização dos modos de existência, tanto em relação aos outros quanto em relação a si mesmo. E, desde que consideremos o sujeito constituído na relação com o outro, devemos considerar que o sujeito ético se constitui por meio de complexas relações intersubjetivas que se dão de acordo com práticas instituídas historicamente (Nardi \& Silva, 2009). Fazer escolhas éticas e políticas, neste contexto, seria poder questionar as verdades instituídas, poder perceber e reposicionar-se nos jogos de poder que estabelecem as verdades de seu tempo - questionar o lugar que se ocupa na relação com o usuário, em relação às escolhas na clínica, em relação aos saberes disciplinares, seus e de seus colegas de trabalho, em relação à gestão e à política que reproduz ou abre brechas no instituído.

Para sustentar uma atitude ética, que exige um movimento intencional em um esforço de transformação, é necessário o reconhecimento explícito dos limites de qualquer saber estruturado, disciplinar (Campos, 2005). Da mesma forma, é necessário reconhecer que a saúde que se almeja é um efeito, uma produção do encontro entre o usuário e os trabalhadores, entre os saberes que cada um carrega consciente e inconscientemente, seus coletivos e o meio em que vivem. Como diz Campos, nas ações sobre o mundo, incluindo aí a clínica, sempre se misturam aspectos do saber, do poder e dos afetos.

$\mathrm{Na}$ narrativa da história de Luís André, os fantasmas, representantes do biopoder, ${ }^{4}$ nos mostraram que não se faz clínica sem política (Pelbart, 2007).

\footnotetext{
${ }^{4}$ Biopoder, conceito cunhado por Foucault (1988), pode ser entendido como formas de ser e de estar no mundo, regidas por um conjunto de preceitos científicos e/ou estéticos que emanam do conjunto de forças que organizam objetiva e subjetivamente a vida contemporânea.
} 
Constroem-se lugares para habitar o campo da Saúde Coletiva, nos quais as escolhas possíveis podem permitir uma infinidade de atitudes. Por exemplo, escolher profanar (Agamben, 2007) os saberes em favor da constituição da autonomia e da ampliação da capacidade de intervenção do sujeito em sua própria vida, como também ocorreu no caso de Luiza. Baseada em uma posição ética e política, a nutricionista transgrediu o instituído para dizer à mãe que, mesmo que Luiza tomasse mamadeira (indicação clínica como complemento alimentar devido à demora em adquirir peso), ela não pararia de mamar ao peito, porque o afeto da amamentação não era substituído pela mamadeira - o que contradiz os saberes científicos e não científicos sobre o tema (Bueno et al., 2002; Figueiredo et al., 2012). Tal posição só se articula em um campo entre saberes, no qual o saber da mãe é colocado em cena. Não há uma hierarquia entre o saber da mãe e o da nutricionista; há, no entanto, a técnica implicada ética e politicamente, como diferença que se faz presente no encontro, com a função de oferecer o cuidado em saúde.

$\mathrm{O}$ momento em que surge a profanação se dá devido à permeabilidade da nutricionista no encontro com a mãe de Luiza. A discussão do caso com a psicóloga, que foi anterior ao encontro, reforçou a permeabilidade do corpo, a capacidade para se deixar afetar, expôr-se, desprender-se de seu próprio saber para

ouvir o saber da mãe. O mesmo ocorreu com a fisioterapeuta que, em determinado momento, conseguiu fazer emergir a posição paterna de Luís Vicente, pai de Luís André. O trabalho em equipe e em rede é fundamental para sustentar uma escolha ético-política e depende de "encantamentos" para se constituir.

\section{Encantamentos}

O termo "encantamento" foi uma escolha que pretendeu explicitar aquilo que Figueiredo (2005) chamou de transferência de trabalho. Trata-se do estabelecimento de um laço produtivo entre os pares, baseado em uma demanda de saber, suposição de saber, que circula em uma equipe com determinada concepção de clínica pautada no sujeito. $\mathrm{O}$ que se compartilha em um trabalho em equipe é o que se recolhe de cada intervenção, aquilo que se colhe no trabalho clínico e com o qual se tece um saber. Trata-se de um saber singular, não só porque se refere a um sujeito singular, aquele que é atendido pelos serviços, mas também porque se trata de um "encantamento" singular. As diferenças entre aqueles que compõem a equipe enriquecem o saber que se constitui coletivamente. Foi a percepção de que seu trabalho seria potencializado na rede que fez os trabalhadores proferirem em conjunto o "encantamento" que criou a Rede Luís André. 
O "encantamento" produz um laço de trabalho a partir do desenvolvimento de um espaço intermediário conjunto: área transicional na qual uma ilusão de suposto saber é compartilhada pela equipe; instância em que seja possível ensaiar decisões coletivas e analisar situações nas quais os trabalhadores estejam implicados (Onocko-Campos, 2012). Os espaços de discussão de caso que aconteciam entre a psicóloga e a nutricionista, no caso de Luiza, e as discussões promovidas pela Rede Luís André ofereciam a função de espaço transicional, uma possibilidade de se implicar no processo e nas decisões do grupo.

Há que se cuidar de uma equipe para que os "encantamentos" produzidos possam ser em favor da produção de possibilidades para a construção de projetos terapêuticos e de vida. Somente quando se está sob forte "encantamento" consegue-se persistir na tarefa árdua de desarticular certezas, apostar na construção de um lugar de sujeito, na força de intervenção sobre a realidade efetuada, nos processos de produção de si e do mundo (Passos \& Barros, 2001).

Trata-se de um plano de coengendramento: a equipe e o cuidado com o usuário se constituem mutuamente. Ambos não nascem prontos, ambos são produzidos pelas formas como as relações se engendram, pelos discursos que promovem a constituição de suas subjetividades e pelo modo como se engajam nestes discursos.

\section{Conclusão}

A narrativa das experiências clínicas na Saúde Coletiva, aqui focada em casos de bebês, pode fornecer importante e delicado material que nos mostra como as relações são tecidas e sustentadas em um espaço no qual o trabalho depende de escolhas éticas e políticas.

As narrativas nos mostraram que Clínica e Saúde Coletiva não formam oposições binárias - como poderíamos pensar desde a história da construção do campo da Saúde Coletiva, quando a clínica foi considerada complementar a este campo (Paim \& Almeida Filho, 1998), produzindo o que Onocko-Campos (2012) chamou de recalcamento da clínica (p. 106). A clínica acontece antes em um movimento entre saberes que circulam em uma superfície paradoxal (Winnicott, 1975), posto que está em movimento e sem definição, precisando ser sustentada, não resolvida. Ocorre uma intercessão (Deleuze, 1992) entre os saberes - um saber causa efeito sobre o outro, acrescentando algo novo em uma relação de suplementaridade (Derrida, 1971). Estabelece-se um movimento de continuidade entre saberes que "torce" a posição que ocupam, como sugere a figura topológica da fita de Moebius proposta por Lacan (1966/1998) — já não é possível definir uma única origem ao saber produzido no encontro. Dessa forma, sugerimos que a relação 
entre Clínica e Saúde Coletiva é da ordem de uma "suplementaridade moebiana" (Torossian, 2009). Que as narrativas clínicas possam contribuir para provocar a emergência de outros olhares sobre a Clínica, a partir da prática que se constitui cotidianamente em Saúde Coletiva, é o que se espera deste trabalho, abrindo um possível campo para novas investigações.

\section{Referências}

Agamben, G. (2007). Profanações. São Paulo: Boitempo.

Benjamin, W. (2012). Magia e técnica, arte e política: ensaios sobre literatura e história da cultura. São Paulo: Brasiliense.

Birman, J. (2005). A physis da saúde coletiva. Physis, 1(1). Recuperado em 25 abr. 2014 de: <http://www.scielo.br/scielo.php?script=sci_arttext\&pi$\mathrm{d}=\mathrm{S} 0103-73311991000100001 \& \operatorname{lng}=\mathrm{en} \& \mathrm{nrm}=\mathrm{iso}>$.

Broide, E.E. (2009). Porosidades clínicas: diálogos entre a psicanálise e a Saúde Coletiva. Boletim da Saúde, 23(2), p. 51-56.

Bueno, M.B. et al. (2002, agosto). Duração da amamentação após a introdução de outro leite: seguimento de coorte de crianças nascidas em um hospital universitário em São Paulo. Revista Brasileira de Epidemiologia, 5(2). Recuperado em 10 fev. 2014 de: <http://www.scielo.br/scielo.php?script=sci_arttex$\mathrm{t} \& \mathrm{pid}=\mathrm{S} 1415-790 \mathrm{X} 2002000200002 \& \operatorname{lng}=\mathrm{pt} \& \mathrm{nrm}=\mathrm{iso}>$.

Campos, G.W.S. (2005). Saúde Paidéia (2a. ed.). São Paulo: Hucitec.

Deleuze, G. (1992). Conversações. Rio de Janeiro: Ed. 34.

Derrida, J. (1971). Gramatologia. São Paulo: Perspectiva.

Derrida, J. (1997). Carta a un amigo japonês. El tiempo de una tesis: desconstrucción y implicaciones conceptuales. Barcelona: Proyectos A Ediciones.

Figueiredo, A.C. (2004, março). A construção do caso clínico: uma contribuição da psicanálise à psicopatologia e à Saúde Mental. Rev. Lat. Am. Psicopat. Fund. VII(1), pp. 75-86. Recuperado em 3 fev. 2014 de: <http://www.fundamentalpsychopathology.org/ uploads/files/revistas/volume07/n1/a_construcao_do_caso_clinico_uma_contribuicao_da_psicanalise_a_psicopatologia_e_a_saude_mental.pdf $>$.

Figueiredo, A.C. (2005, novembro) Uma proposta da psicanálise para o trabalho em equipe na saúde mental. MENTAL - Revista de Saúde Mental e Subjetividade da UNIPAC/ MG, Barbacena, MG, $I I I(5)$, p. 43-55.

Figueiredo, S.F. et al. (2012). Iniciativa Hospital Amigo da Criança: uma política de promoção, proteção e apoio ao aleitamento materno. Acta Paulista de Enfermagem, 25(3). Recuperado em 28 out. 2013 de: <http://www.scielo.br/scielo.php?script=sci_arttext\&pid=S0103-21002012000300022\&lng=en\&nrm=iso $>$. 


\section{SAÚDE MENTAL}

Foucault, M. (1988). História da sexualidade I - Vontade de saber. Rio de Janeiro: Edições Graal.

Jerusalinsky, J. (2002). Enquanto o futuro não vem: a psicanálise na clínica interdisciplinar de bebês. Salvador: Ágalma.

Lacan, J. (1998). Escritos. Rio de Janeiro: Jorge Zahar. (Trabalho original publicado em 1966).

Luz, M. T. (2009). Complexidade do campo da Saúde Coletiva: multidisciplinaridade, interdisciplinaridade, e transdisciplinaridade de saberes e práticas - análise sócio -histórica de uma trajetória paradigmática. Saúde e Sociedade, 18(2), São Paulo. Recuperado em 10 jan. 2014 de: <http://www.scielo.br/scielo.php?script=sci_arttext\&pid=S0104-12902009000200013\&lng=en\&nrm=iso $>$.

Nardi, H.C., \& Silva, R.N. (2009). Ética e subjetivação: as técnicas de si e os jogos de verdade contemporâneos. In N.M.F. Guareschi et al., Foucault e a psicologia (pp. 143-157). Porto Alegre: EDIPUCRS.

Nunes, E.D. et al. (2010, junho). O campo da Saúde Coletiva na perspectiva das disciplinas. Ciência \& Saúde Coletiva, 15(4). Recuperado em 25 abr. 2014 de: <http://www.scielosp.org/scielo.php?script=sci_arttext\&pid=S1413-81232010000400007\&lng $=$ en\&nrm=iso $>$.

Onocko-Campos, R. T. (2012). Psicanálise \& Saúde Coletiva: interfaces. São Paulo: Hucitec.

Paim, J.S., \& Almeida Filho, N. (1998, agosto). Saúde coletiva: uma "nova saúde pública" ou campo aberto a novos paradigmas? Rev. Saúde Pública, 32(4). Recuperado em 20 jan. 2014 de: <http://www.scielo.br/ scielo.php?script=sci_arttext\&pid=S0034-89101998000400001\&lng=en\&nrm=iso $>$.

Palombini, A. et al. (2011). A escrita como cuidado de si: uma oficina com trabalhadores da saúde mental. APPOA, Psicanálise e intervenções sociais. Porto Alegre: Autor.

Passos, E., \& Barros, R. B. (2001). Clínica e biopolítica na experiência do contemporâneo. Psicologia Clínica Pós-Graduação e Pesquisa (PUC/RJ), 13(1), 89-99.

Pelbart, P. P. (2007). Biopolítica. Sala Preta, USP-ECA, 7(1). Recuperado em 22 mar. 2014 de: $<$ http://www.eca.usp.br/salapreta/PDF07/SP07_08.pdf.>.

Rodulfo, R. (2008). El psicoanálisis de nuevo: elementos para la desconstrucción del psicoanálisis tradicional. Buenos Aires: Eudeba.

Tambellini, A.T., \& Schütz, G.E. (2009, setembro/dezembro). Contribuição para o debate do Cebes sobre a "Determinação Social da Saúde": repensando processos sociais, determinações e determinantes da saúde. Saúde em Debate, Rio de Janeiro, 33(83). Recuperado em 18 maio de 2014 de: <http://www.cebes.org.br/media/file/SDv33n83.pdf>

Torossian, S.D. (2007). Uma atitude do pesquisar: psicanálise e pesquisa-intervenção. XIV Encontro Nacional da Abrapso, Rio de Janeiro. Recuperado em 26 abr. 2014 de: $<$ http://www.abrapso.org.br/siteprincipal/anexos/AnaisXIVENA/conteudo/html/ mesa/2195_mesa_resumo.htm\#3> 
Torossian, S.D. (2009). Entre a psicanálise e a saúde coletiva: encontros com a dor, narrativas literárias, vulnerabilidades e infâncias. Boletim da Saúde, 23(2), 101-112.

Winnicott, D.W. (1975). O brincar e a realidade. Rio de Janeiro: Imago.

Reconhecimento: Agradecemos às nutricionistas Daniela Schneider e Mabilda Dotto e à psicóloga Marília Silveira, ex-estagiária do Nutrir, a contribuição como pesquisadoras-colaboradoras na pesquisa de mestrado que deu origem a este artigo.

\section{Resumos}

(Among babies, abysses and ghosts: narratives to reflect on the relation between clinical practice and public health)

This paper reflects on the relation between clinical practice and public health using narratives of clinical cases of babies treated by the Public Health System's Primary Care with the support of the psychoanalytical theory. It was observed that the encounter involving user knowledge, the technical knowledge of the subject matters, and the knowledge of Public Health produced a motion that circulates on a paradoxical 304 surface, producing a relation of "moebian supplementarity" between clinical practice and public health.

Keywords: Clinical practice, public health, early childhood, psychoanalysis

(Parmi bébés, abîmes et les fantômes: des récits pour réfléchir à propos du rapport entre clinique et santé publique)

Cet article propose une réflexion sur le rapport entre la clinique et la santé publique à partir de témoignages de cas cliniques de bébés traités avec l'appui théorique de la psychanalyse dans le cadre des soins de base du Système Unifié de Santé (SUS). Nous avons observé que la rencontre entre les savoirs des usagers, les connaissances techniques des disciplines concernées et les savoirs de la santé publique produit un mouvement qui circule sur une surface paradoxale, produisant un rapport de «supplementarité moebienne» entre la clinique et la santé publique.

Mots clés: Clinique, santé publique, petite enfance, psychanalise

(Entre bebés, abismos y fantasmas: narrativas para pensar en la relación entre clínica y salud colectiva)

El artículo propone una reflexión sobre la relación entre clínica y salud colectiva desde narrativas de casos clínicos de bebés atendidos con un aporte teórico del psicoanálisis en el ámbito de la atención básica del Sistema Único de Salud. Se observó que, en el encuentro entre los saberes de los usuarios, los saberes técnicos de las disciplinas 


\section{SAÚDE MENTAL}

involucradas y los saberes de la salud colectiva, se da un movimiento que circula en una superficie paradojal, produciendo una relación de "suplementariedad moebiana" entre clínica y salud colectiva.

Palabras claves: Clínica, salud colectiva, psicoanálisis

(Unter Babys, Abgründen und Gespenstern: Erzählungen zur Reflektion über die Beziehung zwischen klinischer Betreuung und kollektiver Gesundheit)

Dieser Artikel legt eine Betrachtung über die Beziehung zwischen klinischer Betreuung und kollektiver Gesundheit vor, die aufgrund Erzählungen klinischer Fälle von behandelten Babys und einem theoretischem Beitrag der Psychoanalyse im Bereich der primären Versorgung des brasilianischen öffentlichen Gesundheitssystems (SUS) zustande kam. Es wurde festgestellt, dass bei der Begegnung des Wissens der Nutzer mit dem technischen Wissen der betroffenen Fachgebieten und dem Wissen der kollektiven Gesundheit eine Bewegung entsteht, die auf einer paradoxalen Fläche zirkuliert und eine Beziehung von „Möbius'scher Supplementarität“ zwischen Klinik und kollektiver Gesundheit erzeugt.

Schlüsselwörter: Klinik, kollektive Gesundheit, frühe Kindheit, Psychoanalyse. 的关系)

（肾儿，深渊和幽灵：从通过精神病的表述来思考临床实践和群体健康之间

文章通过对来自贞儿的临床病例叙述进行分析, 反思巴西健康统一系统 (SUS) 的初级门诊(Atenção Básica)中的临床诊和群体健康之间的关系。我们观 察到这样一种现象: 在健康系统使用人的认知(saberes dos usuários)和专业人员的 认知以及群体健康方面的认知的交汇中产生了一种悖论性的层面(superfície paradoxal), 因而产生了临床和群体健康之间的莫比互补(suplementaridade moebiana)。 关键词: 临床, 群体卫生, 婴幼儿期, 精神分析

Citação/Citation: Müller, C.O., \& Palombini, A. de L. (2015, junho). Entre bebês, abismos e fantasmas: narrativas para pensar a relação entre clínica e saúde coletiva. Revista Latinoamericana de Psicopatologia Fundamental, 18(2), 292-306.

Editoras do artigo/Editors: Profa. Dra. Ana Cristina Costa de Figueiredo e Profa. Dra. Andrea Máris Campos Guerra.

Recebido/Received: 3.3.2015/3.3.2015 Aceito/Accepted: 25.3.2015/3.25.2015 
Copyright: (C) 2009 Associação Universitária de Pesquisa em Psicopatologia Fundamental/ University Association for Research in Fundamental Psychopathology. Este é um artigo de livre acesso, que permite uso irrestrito, distribuição e reprodução em qualquer meio, desde que o autor e a fonte sejam citados / This is an open-access article, which permits unrestricted use, distribution, and reproduction in any medium, provided the original authors and sources are credited.

Financiamento/Funding: As autoras declaram não terem sido financiadas ou apoiadas / The authors have no support or funding to report.

Conflito de interesses/Conflict of interest: As autoras declaram que não há conflito de interesses / The authors have no conflict of interest to declare.

\section{Cléudia Odiléia Müller}

Mestre em Psicologia Social e Institucional pela Universidade Federal do Rio Grande do Sul - UFRGS (Porto Alegre, RS, Br).

Rua Confraternização, 341/602 - Bairro Pátria Nova

93410-100 Novo Hamburgo, RS, Br

e-mail: claudiaom@uol.com.br

\section{Analice de Lima Palombini}

Docente do Programa de Pós-Graduação em Psicologia Social e Institucional da Universidade Federal do Rio Grande do Sul - UFRGS (Porto Alegre, RS, Br).

Rua Cel. Paulino Teixeira, 293/8 - Bairro Rio Branco

90420-160 Porto Alegre, RS, Br

e-mail: analice.palombini@ufrgs.br 